\title{
An elderly man with excruciating retrosternal pain and dysphagia
}
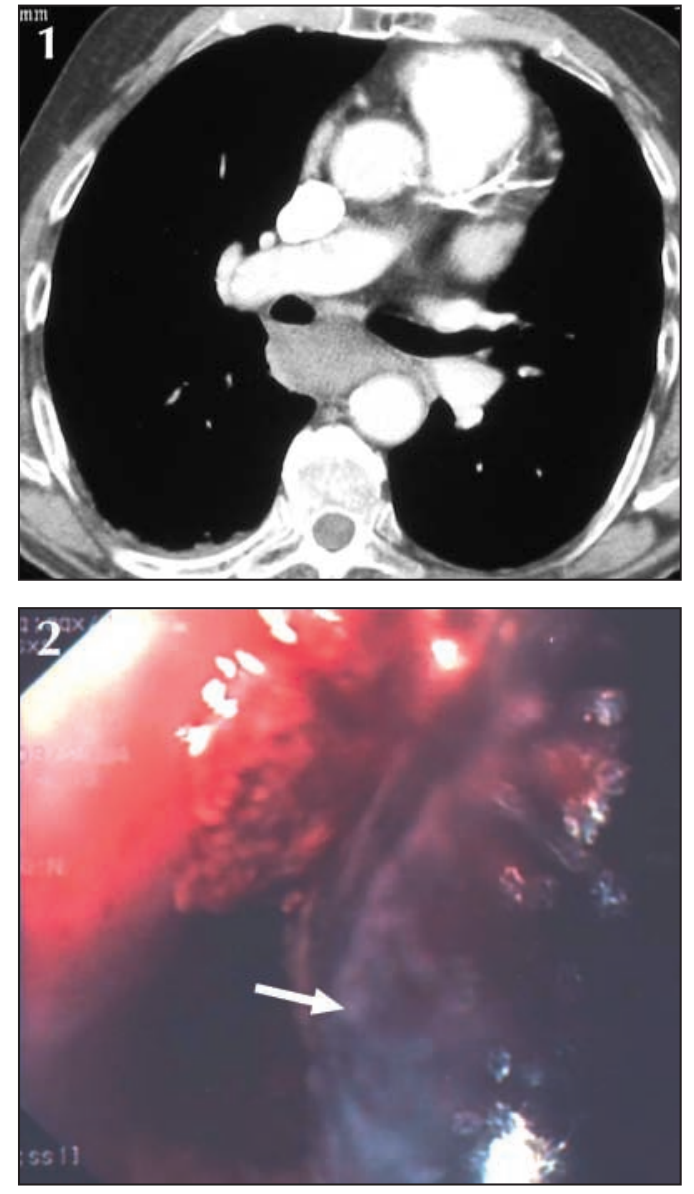

478-year-old man presented A to the emergency department with a 1-day history of progressive, retrosternal chest pain radiating up to his throat that began suddenly upon rising after reaching into the refrigerator. At presentation, the pain had intensified to 9 on a scale of 10 . There was no associated cough or shortness of breath, sweating, nausea, hematemesis or melena. He had a history of hypertension, dyslipidemia and coronary artery disease with left ventricular hypertrophy, diastolic dysfunction, calcific aortic stenosis and paroxysmal atrial fibrillation. He had required right carotid endarterectomy and coronary artery bypass grafting 10 years ago and stenting of one of the grafts 3 months before presentation. His current medications included warfarin, furosemide, simvastatin, mononitrates, ASA and amiodarone.

Other than appearing acutely ill, his findings on examination, including his vital signs, were normal. ASA and nitroglycerin spray had no effect on his pain. Blood tests revealed mild anemia (hemoglobin level 115 [normal 136-172] g/L), an international normalized ratio of 1.9 (normal < 1.22) and a negative troponin result. Electrocardiography showed atrial fibrillation (heart rate about 70 beats/min) with no ischemic changes, and a chest radiograph was normal.

A chest CT scan ordered to rule out a dissecting aortic aneurysm instead revealed an eccentric thickening of the esophageal wall with compression of the lumen (Fig. 1, arrow). In retrospect, the patient had experienced difficulty swallowing since the onset of the pain. Careful endoscopy showed a bluish, submucosal lesion (Fig. 2, arrow) oozing small amounts of blood over one-third of the perimeter of the esophagus at $25 \mathrm{~cm}$ and extending to the stomach. Dissecting intramural esophageal hematoma was diagnosed. The patient was started on a treatment of soft diet, pain control and antacids, and warfarin and ASA were stopped. His hospital course was uneventful except for mild melena, and he was discharged home after 7 days.

Dissecting submucosal esophageal hematoma is rare and is usually associated with forceful vomiting, straining, iatrogenic instrumentation or trauma. Timely diagnosis of a spontaneous occurrence may be difficult. Known risk factors include advancing age, the presence of bleeding disorders, and the use of anticoagulant, antiplatelet or thrombolytic drugs. ${ }^{1}$ Classic presentation involves the sudden unprovoked onset and ripping quality of severe retrosternal pain radiating to the neck, which may take several hours to peak and may have a prolonged, intermittent course. The presence of dysphagia or odynophagia is highly suggestive of dissecting submucosal esophageal hematoma. Melena or mild hematemesis may occur. ${ }^{1}$ Physical examination and blood tests are not usually helpful in diagnosis. Spiral CT or MRI are the preferred diagnostic methods, ${ }^{2}$ as they are safer than endoscopy (which is relatively contraindicated) and useful in ruling out other causes of acute chest pain. Management involves pain control, restoring normal coagulation and soft diet to prevent food-related trauma. Complications include upper gastrointestinal bleeding, esophageal rupture (Boerhaave's syndrome) and, rarely, esophageal or tracheal obstruction. Most patients do well with prompt diagnosis and adequate treatment; ${ }^{3}$ however, misdiagnosis as cardiac pain could lead to the potentially fatal use of anticoagulants or thrombolytic agents. ${ }^{1}$

\section{Ami Schattner}

Department of Medicine

Addenbrooke's Hospital

University of Cambridge

Cambridge, UK

Yuval Binder

Ehud Melzer

Department of Gastroenterology

Kaplan Medical Centre

Rehovot, Israel

Hebrew University Hadassah

Medical School

Jerusalem, Israel

\section{References}

1. McGurk C, Rodgers C, Kirby J Tony C, Tham K. An uncommon but important cause of severe chest pain in an older population. Age Ageing 2001;30;357.

2. Hiller N, Zagal I, Hadas-Halpern I. Spontaneous intramuaral hematoma of the esophagus. Am $\mathcal{F}$ Gastroenterol 1999;94:2282-4.

3. McIntyre AS, Ayres R, Atherthon J, Spiller RC, Cockel R. Dissecting intramural haematoma of the oesophagus. Q7M 1998;91:701-5. 